\title{
Intramolecular Contributions to Stretched-Exponential Relaxation Behavior in Polymers
}

\section{Ivet Bahar* and Burak Erman}

Polymer Research Center, School of Engineering, Bogazici University, and TUBITAK Advanced Polymeric Materials Research Center, Bebek 80815, Istanbul, Turkey

\author{
George Fytas \\ Research Center of Crete, P.O. Box 1527, 71110 Iraklion, Crete, Greece \\ Werner Steffen \\ Max-Planck-Institut für Polymerforschung, P.O. Box 3148, D-55021 Mainz, Germany \\ Received January 27, 1994; Revised Manuscript Received June 9, $1994^{\circ}$
}

\begin{abstract}
Theoretical and experimental evidence for the occurrence of multiple relaxation regimes in polymers far above the glass transition temperature is presented and interpreted in terms of the KohlrauschWilliams-Watts (KWW) stretched exponential expression. Intramolecular effects responsible for the observation of stretched exponential decay of correlation functions are discussed in relation to four different theoretical models: The kinetic Ising model of Glauber, the Shore-Zwanzig model of linear fluctuating dipoles, the Hall-Helfand model of bistable oscillators, and the dynamic rotational isomeric state model. A decrease in the $\mathrm{KWW}$ exponent is predicted with increasing intramolecular coupling. Chain connectivity appears as a major factor underlying the stretched exponential behavior observed in an intermediate time regime covering a time span of 2-3 orders of magnitude. In the short time limit, a single exponential decay of correlations is approached in all models. At long times, on the other hand, different $\mathrm{KWW}$ exponents are observed depending on the degree of intramolecular cooperativity. Results of depolarized Rayleigh scattering measurements by Tandem Fabry-Perot interferometry on poly(methylphenylsiloxane) at high temperatures are observed to be insensitive to variations in temperature and concentration, which lends support to the notion that intramolecular interactions play a fundamental role in ascribing the apparent KWW behavior.
\end{abstract}

\section{Introduction}

Dielectric relaxation, quasielastic light scattering, and viscoelastic measurements of local segmental dynamics in bulk polymers ${ }^{1-4}$ indicate that the time decay of correlation functions $C(t)$ conforms, to a good approximation, with the empirical Kohlrausch-Williams-Watts (KWW) stretched exponential expression $^{5}$

$$
C(t)=\exp \left[-(t / \tau)^{\beta}\right]
$$

Here $\tau$ is the characteristic correlation time associated with $C(t)$. The exponent $\beta$ is viewed as a measure of cooperativity, decreasing from 1 , in the case of independent units, to lower values, in the presence of coupling between relaxing units. Experiments yield a value of $\beta$ varying between 0.3 ard 0.45 for amorphous polymers above the glass transition temperature.

The correlation function $C(t)$ is expressed as the ensemble average

$$
C(t)=\langle X(t) X(0)\rangle
$$

where $X(0)$ is, in general, the dynamic or stochastic variable at time $t=0$, which assumes the value $X(t)$ at time $t$. The averaging is carried out over all possible conformations of the dynamic variable at times zero and $t$. In dielectric spectroscopy, for example, $X(t)$ is the dipole moment while in depolarized Rayleigh scattering, $X(t)$ is a suitable component of the anisotropic polarizability tensor.

Both intermolecular and intramolecular correlations might be responsible for the experimentally observed stretched exponential behavior. Evidence for the effect of intermolecular correlations is provided by depolarized dynamic light scattering studies on molecular glass formers

- Abstract published in Advance ACS Abstracts, July 15, 1994. such as 0 -terphenylyl and dioctyl phthalate near and above the glass transition temperature $T_{\mathrm{g}}{ }^{6,7}$ The time decay of the scattering function for these materials obeys the $\mathrm{KWW}$ form with $\beta$ less than unity, ${ }^{7-10}$ in agreement with results from dielectric relaxation measurements. ${ }^{11}$ At temperatures far above $T_{\mathrm{g}}\left(T>T_{\mathrm{g}}+80 \mathrm{~K}\right)$ at which fast orientation processes take place, the experimentally obtained orientation correlation function becomes indistinguishable from a single Debye $(\beta=1)$ relaxation, and the temperature dependence of $\tau$ conforms to an Arrhenius equation. For macromolecular glass formers, on the other hand, $C(t)$ measured by dielectric spectroscopy 2,3 (DS) and depolarized Rayleigh spectroscopy ${ }^{12}$ (DRS) far above $T_{\mathrm{g}}$ appears to retain its nonexponential shape which is observed near $T_{\mathrm{g}}$. Apparently, this different behavior between molecular and polymeric viscoelastic liquids well above $T_{\mathrm{g}}$ is due to the intramolecular contributions to $C(t)$ of the latter class of materials, because at these temperatures free volume and hence intermolecular effects loose their importance. Experimental evidence of intramolecular effects on $C(t)$ also originates from time resolved optical spectroscopic measurements of labeled polyisoprene solutions ${ }^{13}$ and recent DRS spectra of a poly(phenylmethylsiloxane) and (PMPS) $/ \mathrm{CCl}_{4}$ solution with 80 wt \% PMPS.

Among various factors affecting chain dynamics, the essential physical feature which needs to be recognized, and in fact forms the basis of most of mathematically tractable models, is the chain connectivity. Interdependence between bonded units, from the point of view of both spatial and conformational energetic requirements, is intrinsically present in polymer chains. It might be interesting to verify whether a stretched exponential behavior is compatible with simple models in which all chain characteristics other than chain connectivity are ignored. One-dimensional models, devoid of any chemical 
and structural constraints, might be a good starting point for exploring the intrinsic role of chain connectivity, if any, on the observed stretched exponential behavior. Models including the three-dimensional structural and conformational characteristics of real chains might be explored, as a next step, for an assessment of the influence of specific intramolecular interactions on chain dynamics. This type of analysis would provide information on the contribution of single chain properties on the time decay of correlation functions. The understanding of the role of intermolecular effects, on the other hand, necessitates the consideration of environmental constraints on chain dynamics, as well as the coupling between external and internal modes of relaxation, and will not be taken up here.

\section{Models and Calculations}

The division of the relaxation function into separate regimes along the time axis is indeed predicted by several models of the isolated chain with intramolecular correlations. In the present work, the predictions of four such models will be discussed. These models are (i) the onedimensional model of Shore and Zwanzig for perpendiculardipole polymers, ${ }^{14}$ (ii) the kinetic Ising model of Glauber, ${ }^{15}$ (iii) the one-dimensional model chain with bistable bond potentials developed by Hall and Helfand, ${ }^{16}$ and (iv) the dynamic rotational isomeric state model of Bahar et al..$^{17}$ These models differ in their physical and mathematical approaches, as will be described below. Yet, they have the following common features: Except for the model of Shore and Zwanzig in which dipolar spins interact with external fields, they are based on the behavior of isolated single chains, and chain units therein are assigned discrete isomeric states. Transitions between these discrete states constitute the basic mechanism of relaxation. A master equation formalism is adopted for the description of conformational stochastics in models ii-iv. In model $i$, on the other hand, the time evolution of the distribution functions is governed by the rotational diffusion equation. Interdependence of bonds along the chain is limited to nearest neighbors, only, in all cases. Thus, these may be identified as Markov chains, in the sense that the dynamic behavior of a given unit depends on the state of its first neighbor along the chain and is not affected by any topological neighbors or by any long-range interactions, other than chain connectivity. Intramolecular correlations, though restricted to first neighbors along the chain, are incorporated at different levels of approximation, in these models. Models i-iii are one-dimensional and yet, capture the essential features of local dynamics. Model iv incorporates the three-dimensional structural characteristics of real chains, by following the rotational isomeric state approximation of chain statistics. A brief description of these models will be given below, and the correlation functions obtained from each model will be evaluated and discussed in relation to the KWW expression.

(i) One-Dimensional Model of PerpendicularDipole Polymers. ${ }^{14}$ This model describes the small amplitude oscillations of dipoles $\mu$ along a linear chain. The dipoles are represented by spins perpendicular to the chain axis, undergoing harmonic interactions with each other and a cosine interaction with the external electric field $E$. The time-dependent behavior of the $N$ dipoles along the chain is described by the autocorrelation function

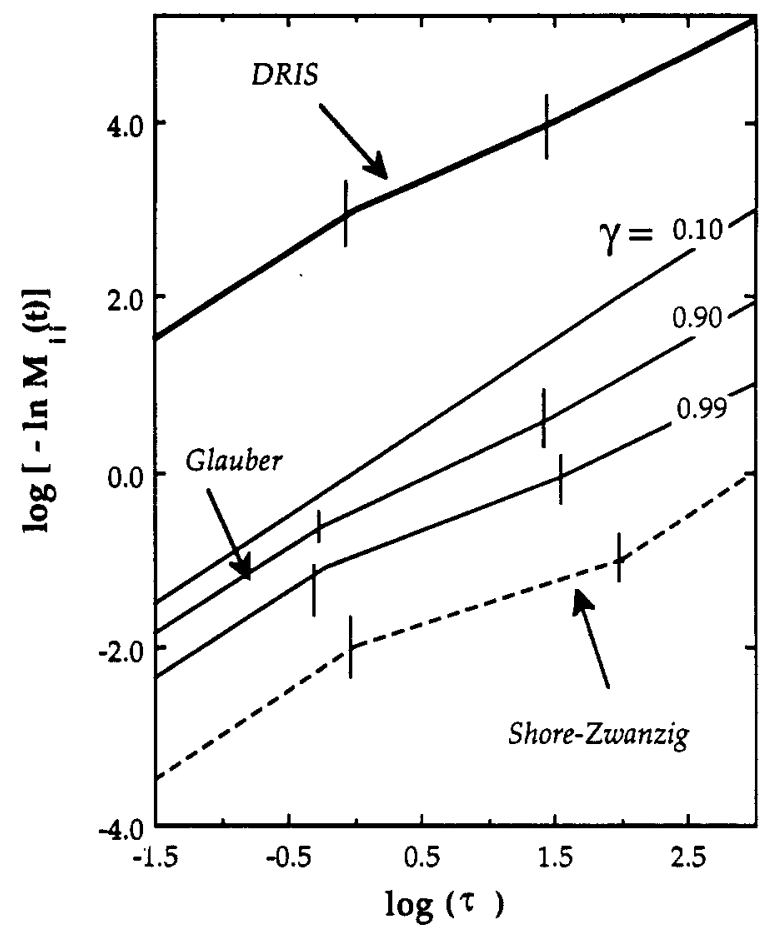

Figure 1. KWW plots of the correlation functions predicted by (i) the Shore-Zwanzig model, (ii) the Glauber model with various coupling parameters $\gamma$ as indicated in the middle three curves, and (iii) the DRIS model, indicating all the occurrences of the three distinct relaxation regimes.

$M(t)$ of the electric moment ${ }^{14}$

$M(t)=\frac{\mu^{2} E}{2 k_{\mathrm{B}} T} \exp \left[-D \int_{0}^{\mathrm{t}} \mathrm{d} s \mathrm{e}^{-2 J D \mathrm{~s} / k_{\mathrm{B}} T} I_{0}\left(2 J D \mathrm{~s} / k_{\mathrm{B}} T\right)\right]$

Here, $k_{\mathrm{B}}$ is the Boltzmann constant, $T$ is the temperature, $D$ is the rotational diffusion coefficient for the dipoles, $J$ is the coupling constant between the neighboring dipoles, and $I_{0}$ is the zeroth order modified Bessel function. The quantity $\mathrm{e}^{-\tau} I_{0}(\tau)$ is pointed out ${ }^{14}$ to appear as a general attribute of one-dimensional stochastic problems with nearest-neighbor interactions, as previously demonstrated by Oppenheim, Shuler, and Weiss for random walks of small particles on discrete, one-dimensional, infinite lattices. ${ }^{18}$ Thus, the diffusion of the orientation of a dipole along the chain, through the mechanism of orientation exchange with its nearest neighbors, is represented by the modified Bessel functions. The normalized decay function $C(t) \equiv M(t) / M(0)$ resulting from eq 3 may be approximated by three simple functions for the short, intermediate, and long time ranges as

$$
\begin{aligned}
C(t) \equiv & \frac{M(t)}{M(0)}= \\
& \begin{cases}\mathrm{e}^{-D t} & \text { for } \mathrm{JD} t / k_{\mathrm{B}} T \ll 1 \\
\exp \left[-\left(\mathrm{D} t k_{\mathrm{B}} T / \pi J\right)^{1 / 2}\right] & \text { for } 1 \ll \mathrm{JD} t / k_{\mathrm{B}} T \ll N^{2} \\
\mathrm{e}^{-\mathrm{D} t / N} & \text { for } N^{2} \ll \mathrm{JD} t / k_{\mathrm{B}} T\end{cases}
\end{aligned}
$$

When represented in terms of the stretched exponential form of eq 1 , these three expressions yield exponents $\beta$ equal to $1,1 / 2$, and 1 for short-, intermediate-, and longtime relaxations, respectively. This behavior is illustrated by the dashed lines in Figure 1, drawn for arbitrary values of the parameters of eq 4 .

The following qualitative interpretation ${ }^{14}$ of eq 4 is given: For short times, the orientation of the dipoles decays 
without interacting with neighbors, resulting in a single exponential decay with time constant $1 / D$. At intermediate times, intrachain harmonic coupling between nearest dipoles results in a cooperative relaxation, and a nonexponential decay function is obtained. At long times, the intramolecular vibrations are equilibrated and the combined motion of all spins, which is slowed down by a factor of $N$ compared to that of a single free spin, is observed.

It is to be noted that the Shore-Zwanzig model is analytically soluble only because the dipoles interact parabolically. A more recent model introduced by Cook and Helfand considers instead the nearest-neighbor interaction potential with $r$ stable states circularly distributed. ${ }^{19}$ This is a more realistic model for simulating rotameric transitions in real polymer chains. However, the conformational correlation functions resulting from this model obey a single exponential time decay corresponding to the long-time behavior of the Shore-Zwanzig model.

(ii) Kinetic Ising Model of Glauber. ${ }^{15}$ This model is based on the assumption that the spins of a linear array of $N$ individual particles are stochastic functions of time $\sigma_{i}(t)(i=1, \ldots, N)$ and take on values of either +1 or -1 . Random transitions between these two states result from the interaction of the spins with an external agency. The presence of the external agency is implicitly assumed, but the mechanism through which it affects the chain is not relevant to the formulation. Intramolecular correlations are introduced by choosing the transition probabilities for any one spin to depend on the state of the first neighboring spins.

The master equation governing the time evolution of the system is given by the expression

$$
\begin{aligned}
\frac{\mathrm{d}}{\mathrm{d} t} p\left(\sigma_{1}, \ldots, \sigma_{N} t\right)=-[ & {\left[\sum_{i} w_{i}\left(\sigma_{i}\right)\right] p\left(\sigma_{1}, \ldots, \sigma_{N} t\right)+} \\
& {\left[\sum w_{i}\left(-\sigma_{i}\right)\right] p\left(\sigma_{1}, \ldots,-\sigma_{i}, \ldots, \sigma_{N} t\right) }
\end{aligned}
$$

Here, $p\left(\sigma_{1}, \ldots, \sigma_{N} t\right)$ is the probability of having state $\sigma_{1}$ for the first spin, $\ldots, \sigma_{N}$ for the $N$ th spin at time $t . w_{i}\left(\sigma_{i}\right)$ is the rate of transition of the ith spin form $+\sigma_{i}$ to $-\sigma_{i}$ while the others remain fixed. The first summation on the righthand side of eq 8 gives the rate of disappearance of the conformation $\left\{\sigma_{1}, \ldots, \sigma_{N}\right\}$; the second term accounts for the creation of this state by the transition of each spin $i$ from the state $-\sigma_{i}$ to $+\sigma_{i}$. Equation 5, written for each of the $2^{N}$ accessible configurations of the spins, may be conveniently organized in matrix notation as $\mathrm{dp} / \mathrm{d} t=\mathbf{A p}(t)$, where $\mathbf{p}(t)$ denotes the array of the probabilities of the $2^{N}$ states and $\mathbf{A}$ is referred to as the transition rate matrix.

The coupling between first neighbors is introduced by choosing the transition rates $w_{i}\left(\sigma_{i}\right)$ to be of the form (1/ 2) $\alpha\left\{1-(1 / 2) \gamma \sigma_{i}\left(\sigma_{i-1}+\sigma_{i+1}\right)\right\}$ which becomes equal to $\alpha / 2$ if the neighboring spins are antiparallel, $\alpha(1-\gamma) / 2$ if the $i$ th spin is parallel to both of its neighbors, and $\alpha(1+\gamma) / 2$ if it is antiparallel. Thus, the parameter $\gamma$ is a measure of the tendency of the spins to align parallel to each other. Parallel alignments of neighboring spins are favored by positive values of $\gamma$, and opposite alignments by negative values. The degree of coupling between neighboring spins increases as $|\gamma|$ approaches unity, which is its maximum allowable value. $\gamma$ is related to the energy of alignment, $J$, as $\gamma=\tanh \left(2 J / k_{\mathrm{B}} T\right)$.

The spin correlation function $C(t) \equiv M_{i k}(t)$ between the $i$ th and $k$ th spins is obtained ${ }^{15}$ for a system in thermal
Table 1. Best Fitting KWW Parameters for Correlation Function of the Kinetic Ising Model

\begin{tabular}{cccccccc}
\hline & \multicolumn{3}{c}{$\beta$} & \multicolumn{4}{c}{$\tau$} \\
\cline { 2 - 3 }$\gamma$ & short & intermediate & long & short & intermediate & long \\
\hline 0.100 & & 0.988 & & & 1.01 & \\
0.900 & 0.96 & 0.73 & 0.85 & 2.68 & 4.28 & 5.64 \\
0.990 & 0.98 & 0.60 & 0.73 & 8.33 & 43.0 & 41.3 \\
0.999 & 0.94 & 0.56 & 0.58 & 34.0 & 462.0 & 395.4
\end{tabular}

equilibrium at temperature $T$, as

$$
\begin{aligned}
M_{i k}(t)=\left\langle\sigma_{i}(0) \sigma_{k}(t)\right\rangle & = \\
& \mathrm{e}^{-\alpha t} \sum_{m=-\infty}^{\infty}\left[\tanh \left(J / k_{\mathrm{B}} T\right)\right]^{|i-k+m|} \mathrm{I}_{m}(\gamma \alpha t)
\end{aligned}
$$

Here, $I_{\mathrm{m}}$ is the modified Bessel function of order $m$. Autocorrelation decay functions calculated using eq 6 for three different values of the intramolecular coupling parameter $\gamma$ are presented in Figure 1. For each value of $\gamma$, eq 6 is evaluated at closely spaced times and the points obtained in this manner are joined by least squares straight lines. For values of $\gamma$ approaching unity, the curves are best fitted by three consecutive straight lines, each yielding the exponents and correlation times listed in Table 1 . At low $\gamma$ values, the three regimes are not distinguishable, and a Debye behavior is observed. The vertical lines in the figure separate the regions with different slopes. Inasmuch as the $\mathrm{KWW}$ exponent $\beta$ gives an indication of the mechanism of relaxation in different time regimes, we may infer from the tabulated data that the initial relaxation process is relatively little affected by intramolecular correlations, $\beta$ values remaining in the vicinity of unity at short times. The intermediate times, on the contrary, are strongly affected by the degree of intrachain coupling, and $\beta$ seems to approach 0.5 , the value predicted by the Shore-Zwanzig theory, as the interdependence between nearest neighbors is sufficiently strong. The terminal region exhibits slightly larger $\beta$ values, gradually merging to the intermediate region behavior with increasing $\gamma$.

In the absence of correlations between spins in the initial state, the conformational autocorrelation function $M(t)$ for a single spin reduces to

$$
M(t)=\mathrm{e}^{-\alpha(1-\gamma) t} \mathrm{e}^{-\alpha \gamma t} I_{0}(\alpha \gamma \mathrm{t})
$$

which readily follows from eq 6 , by taking $i=k$ and $m=$ 0 . Here, the product $\mathrm{e}^{-\alpha \gamma t} I_{0}(\alpha \gamma t)$ represents the probability for a given spin to remain in its initial state at time $t$.

(iii) Hall-Helfand Model Chain of Bistable Bonds. ${ }^{16}$ This model has been successful in describing experimental data on local relaxation. It is a one-dimensional chain whose bonds may be in either of two states, short (s) and long (l). The state of a given bond $k$ is conveniently denoted by $\sigma_{k}$, assuming the values of +1 or -1 , depending on the state, $s$ or 1 , of that bond. Accordingly, $2^{N}$ configurations, denoted as $\left\{\sigma_{i}=\left\{\sigma_{1}, \ldots, \sigma_{k}, \ldots, \sigma_{N}\right\}_{i}, 1 \leq i\right.$ $\leq 2^{N}$, are accessible to a chain of $N$ bonds, and transitions between those states are governed by a master equation, of the same form as eq 5. Only single step transitions are possible according to the model, those occurring between configurations differing by (i) the state of a single bond or (ii) the states of two adjacent bonds which are themselves of opposite states. These two types of transitions are schematically shown as $\mathrm{s} \leftrightarrow \mathrm{l}$ and $\mathrm{sl} \leftrightarrow \mathrm{ls}$, respectively. The former is referred to as an isolated transition and involves a relatively slow relaxation rate $\lambda_{0}$ inasmuch as it entails 


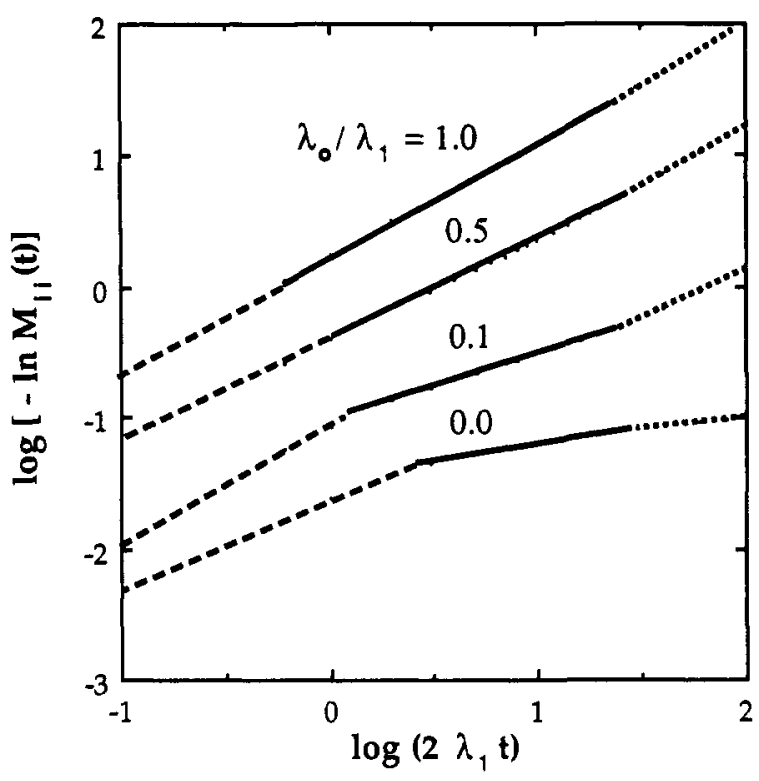

Figure 2. KWW plots of the conformational correlation functions predicted from the Hall-Helfand model for various values of the ratio $\lambda_{0} / \lambda_{1}$ indicated on the figure. The correlation functions are approximated by three consecutive stretched exponentials, with respective exponents listed in Table 2.

Table 2. Best Fitting KWW Parameters for the Hall-Helfand Correlation Function

\begin{tabular}{cccc}
\hline & \multicolumn{3}{c}{$\beta$} \\
\cline { 2 - 4 }$\lambda_{0} / \lambda_{1}$ & short & intermediate & long \\
\hline 1.00 & 0.954 & 0.850 & 0.949 \\
0.50 & 0.937 & 0.756 & 0.904 \\
0.10 & 0.912 & 0.497 & 0.674 \\
0.01 & 0.903 & 0.325 & 0.289 \\
0.00 & 0.850 & 0.274 & 0.174
\end{tabular}

the displacement of the tails and thus requires a largescale spatial reorganization of the chain. The second involves two compensating transitions localizing the motion and, hence, is referred to as a correlated transition; its rate $\lambda_{1}$ is higher than $\lambda_{0}$. The conformational autocorrelation function for the state of a given bond is obtained by the use of Pauli spin operators as ${ }^{16}$

$$
\begin{aligned}
C(t)=M_{k k}(t) \equiv\left\langle\sigma_{k}(0) \sigma_{k}(t)\right\rangle= \\
\quad \exp \left(-2 \lambda_{0} t\right) \exp \left(-2 \lambda_{1} t\right) I_{0}\left(2 \lambda_{1} t\right)
\end{aligned}
$$

This form suggests that $\lambda_{1}$ governs the rate of diffusion or propagation of conformational motion along the chain, whereas $\lambda_{0}$ is responsible for the loss of conformational states. It is interesting to note that eq 7 reduces to eq 6 with the following assignment of parameters:

$$
\alpha=2\left(\lambda_{0}+\lambda_{1}\right) \quad \gamma=\frac{\lambda_{1}}{\lambda_{0}+\lambda_{1}}
$$

Results obtained with eq 7 are shown in Figure 2 for various values of the ratio $\lambda_{0} / \lambda_{1}$. This ratio may be envisaged as a measure of cooperativity, decreasing with the extent of cooperative transitions. The limiting value of $\lambda_{0} / \lambda_{1}=0$ corresponds to the extreme case where all conformational passages occur in the form of correlated transitions. The curves are suitably approximated by three consecutive stretched exponentials, with respective exponents listed in Table 2. In parallel with the results obtained by the Glauber model, an approximately single exponential behavior is observed at short times, followed by a stretched exponential time decay at intermediate times. At long times, the exponent decreases with decreasing $\lambda_{0} / \lambda_{1}$. The three regimes, displayed by the dashed, solid, and dotted lines in the figure, are particularly distinct in the cases $\lambda_{0} / \lambda_{1}=0.1$ and 0.5 , whereas for $\lambda_{0} / \lambda_{1}=1$ the exponents in the different regimes are hardly distinguishable.

(iv) Dynamic Rotational Isomeric State (DRIS) Model. ${ }^{17,20-23}$ The three-dimensional structural and conformational characteristics of real chains and nearestneighbor interactions along the chain are rigorously considered in this model. Here, the torsional rotations $\phi_{i}$ of internal bonds, with indices $1 \leq i \leq N$, are the only structural parameters defining a given chain configuration. Discrete values corresponding to rotational isomeric minima are adopted for torsional angles and fluctuations about the energy wells are neglected. For the case of $\nu$ rotameric states accessible to each bond, a total of $\nu^{N}$ configurations $\{\sigma\}_{m}$ is accessible. $\{\sigma\}_{m}$ is given by a sequence of dihedral angles $\left\{\phi_{1}, \phi_{2}, \ldots, \phi_{N-1}, \phi_{N}\right\}_{m}$, corresponding to the torsional states of the bonds, with $1 \leq m \leq \nu^{N}$. A master equation formalism, like models ii and iii, is adopted for describing the stochastics of configurational transitions between the $\nu^{N}$ configurations. The solution of the master equation leads to correlation functions of the form ${ }^{21}$

$$
C(t)=\sum_{m} k_{m} \exp \left\{\lambda_{m} t\right\}
$$

Here $\lambda_{m}$ is the $m$ th eigenrate of the transition rate matrix A of the DRIS model and $k_{m}$ is the amplitude factor which is a function of the eigenvectors, the configurational property under consideration, and the chain equilibrium statistics. According to eq 9 , a distribution of relaxational modes, with frequencies equal to $-\lambda_{m}, 1 \leq m \leq \nu^{N}$, contributes additively to $C(t)$.

The degree of correlation between the initial and final rotational states of a given bond $i$ may be investigated by considering the product $\left\{\phi_{i}\right\}_{m}\left\{\phi_{i}\right\}_{n}$ where $\left\{\phi_{i}\right\}_{m}$ denotes the state of the $i$ th bond in the $m$ th configuration of the chain. Using the DRIS formalism, the conformational autocorrelation function $M_{i i}(t)$ has been calculated for polyethylene bonds which are subject to the rotational states trans $(\mathrm{t})$, gauch $e^{+}\left(\mathrm{g}^{+}\right)$, and gauche ${ }^{-}(\mathrm{g})$. Details of calculations may be obtained from refs 17 and 20-23. Results are presented as the upper boldface curve in Figure 1. This curve is obtained by adopting the arbitrary value $\left\{\phi_{i}\right\}_{m}=$ 1 for $i=$ trans, and $\left\{\phi_{i}\right\}_{m}=-(1 / 2) P(\mathrm{t}) / P\left(\mathrm{~g}^{ \pm}\right)$for $i=$ gauche $^{ \pm}$, with the energy parameters for real polyethylene chains given in ref 24 . The horizontal and the vertical positions of the curve in the figure are arbitrarily chosen. Three regions with respective slopes $0.98,0.66$, and 0.78 emerge, in parallel with the conformational autocorrelations resulting from one-dimensional models. For independent bonds, a line with a single slope $\beta=1.00$ is obtained for all time regimes, which verifies that a single exponential relaxation occurs in the absence of neighbor dependence.

As a final remark, we note that the DRIS formalism has been recently applied to the relaxation of $c i s$-polyisoprene in the bulk state, and the time decay of dielectric relaxation functions has been analyzed in terms of KWW representation. ${ }^{4}$ Short and intermediate regime behaviors with respective exponents of 0.96 and 0.62 were theoretically predicted when coupling along the chain was restricted to first neighbors only. Upon consideration of a cooperative relaxation mechanism involving larger size chain segments, the KWW exponent was found to decrease to $\sim 0.35$, in good agreement with experiments. Yet, the theoretically predicted time range for intermediate regime behavior was narrower than that experimentally observed. Fluctuations of the environment were invoked for extending 


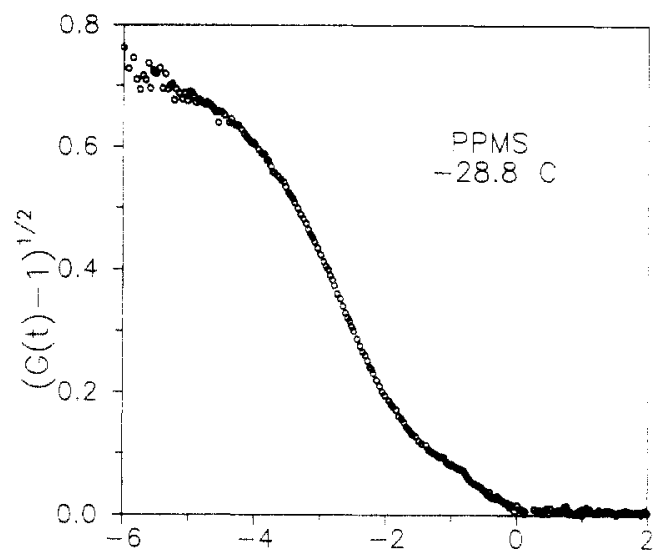

Figure 3. Orientation time correlation function $C(t)=[G(t)-$ $1]^{1 / 2}$ of bulk poly(methylphenylsiloxane) at $244.4 \mathrm{~K}$. The depolarized intensity correlation function $G(t)$ was recorded by a digital full ALV-5000 correlator.

the KWW behavior to time intervals of about four decades, in conformity with experiments. ${ }^{4}$

\section{Experimental Evidence of Nonexponential Behavior Due to Intramolecular Contributions}

As previously mentioned, segmental orientation time correlation functions $C(t)$ (eq 2) can be conveniently measured by the DRS technique. For slow correlation times $\left(\tau>10^{-6} \mathrm{~s}\right)$ near $T_{\mathrm{g}}$, one can employ photon correlation spectroscopy (PCS) whereas for faster motions taking place far above $T_{g}$ Fabry-Perot interferometry (FPI) can be utilized to record the spectrum $I_{\mathrm{VH}}(\omega)$. The latter is the Fourier transform of $C(t)$. In principle, $C(t)$ measured by DRS reflects collective orientation. It is known $^{12}$ that intermolecular orientational correlations are weak in amorphous bulk homopolymers such as PMPS. On the other hand, the extent of intramolecular correlations is not experimentally well established yet. Here, we present both PCS and FPI-DRS measurements on amorphous PMPS $\left(M_{\mathrm{w}}=5 \times 10^{3}\right)$ aiming mainly at the shape of $C(t)$ and its relation to intramolecular effects.

Figure 3 shows an experimental $C(t)$ of PMPS measured by PCS at $244.4 \mathrm{~K}$. The ordinate $[G(t)-1]^{1 / 2}$ in the figure corresponds directly to $C(t)$. In the case of the common KWW type relaxation, this time correlation function is described by the parameters $\tau$ and $\beta$ and the amplitude. In the present case, however, a single KWW representation is not adequate and displays systematic deviations. In fact, a second slower relaxation process with $\beta \approx 1$ should be included in the fitting procedure in order to adequately describe the experimental $C(t)$. This virtually Debye slow relaxation mode with correlation times over 100 times longer than $\tau$ could be associated with the slow decays of Figures 1 and 2 which reflect global chain orientational motions. The main relaxation process for PMPS in Figure 3 exhibits a KWW exponent of $\beta=0.44 \pm 0.02$.

The nonexponential decay of $C(t)$ commonly observed near and above $T_{\mathrm{g}}$ has been ascribed to regions subject to cooperative rearrangements with lifetime $\tau .{ }^{25,26}$ The size of these regions decreases with increasing temperature, thus accounting for the concomitant increase of $\beta$ in molecular glass formers. 6,7 In fact, although molecular glass forming liquids show $\beta<1$ at low $T$ near $T_{\mathrm{g}}$, the exponent $\beta$ becomes indistinguishable from unity at $T>$ $T_{\mathrm{g}}+80 \mathrm{~K}^{6,7,27-29}$ It appears, however, that amorphous polymers do not conform with this scheme. ${ }^{12,30}$ We have recorded the $I_{\mathrm{VH}}(\omega)$ spectra of PMPS at high temperatures by using for the first time Tandem FPI in order to diminish the neighboring interferometric orders. At $T=353 \mathrm{~K}$,
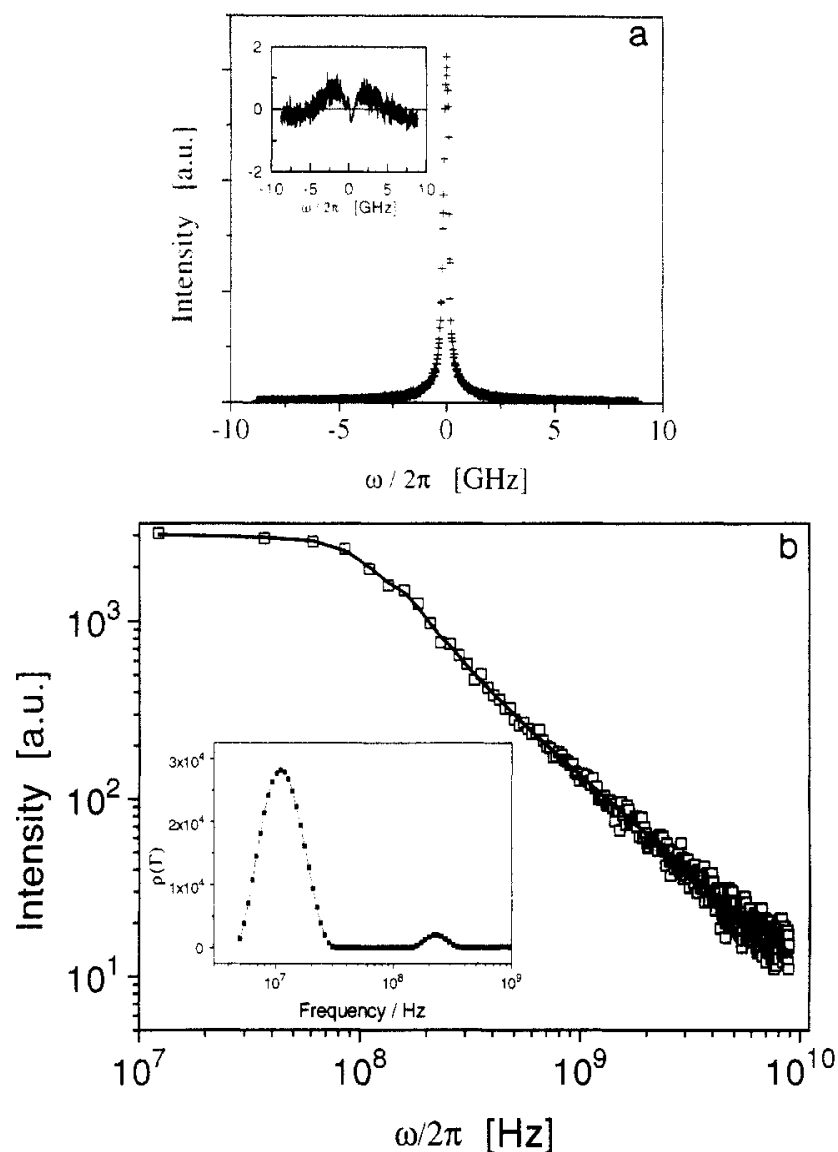

Figure 4. (a) Depolarized Rayleigh spectrum $I_{\mathrm{VH}}(\omega)$ of bulk poly(methylphenylsiloxane) at $353 \mathrm{~K}$ recorded by a tandem Fabry-Perot interferometer at a free spectral range of $10 \mathrm{GHz}$. The convolution of a single Lorentzian line with the instrumental function provides a poor representation of the experimental spectrum, as shown in the deviation plot (inset). (b) Fit of a continuous distribution of Lorentzian line shapes convoluted with the instrumental function to the experimental $I_{\mathrm{VH}}(\omega)$ respectively denoted by the solid line and the squares. The obtained distribution $p(\Gamma)$ is shown in the inset.

about 120 deg above $T_{\mathrm{g}}$, the experimental $I_{\mathrm{VH}}(\omega)$ displayed in Figure 4a cannot be adequately described by a single Lorentzian $(\beta=1)$ line convoluted with the instrumental function, as is evident from the deviation plot (inset of Figure 4a). Instead, the transformation of $I_{\mathrm{VH}}(\omega)$ spectra of Figure 4a to the time domain, and subsequent fit to a single KWW led to $\beta=0.50 \pm 0.10$, which is close to the exponent observed at $244.4 \mathrm{~K}$. It should be pointed out that the differentiation between a double Lorentzian and a single KWW fit of $I_{\mathrm{VH}}(\omega)$ is somewhat ambiguous, as described in detail in ref 30 . In contrast, the PCS functions (Figure 3 ) are much more amenable to line shape studies. Indeed, the integrated intensities $I_{\mathrm{VH}}$ are found to be insensitive to temperature variations between 290 and 343 $\mathrm{K}$ for bulk PMPS. It is to be noted that for bulk polyisoprene and polybutadiene $I_{\mathrm{VH}}$ was also observed to be a weak function of temperature (merely density effects) over the same range. ${ }^{30}$

In parallel with the increase of temperature, dilution of PMPS by addition of $20 \mathrm{wt} \% \mathrm{CCl}_{4}$ (optically isotropic solvent) has no measurable effect on $I_{\mathrm{VH}}(\omega)$ other than reduction of the integrated intensities by also $20 \%$. The insensitivity of the shape of $I_{\mathrm{VH}}(\omega)$ to temperature and concentration variations corroborates the notion that intramolecular effects, rather than intermolecular, are the source of the observed behavior. Thus, as discussed in the previous section, intramolecular correlations play a significant role in the relaxation mechanism, at least at 
high temperatures.

The $I_{\mathrm{VH}}(\omega)$ spectrum of Figure $4 \mathrm{a}$ has been analyzed by a continuous distribution $\rho(\Gamma)$ of Lorentzians with relaxation rate $\Gamma$ after convolution with the instrumental function. As described elsewhere, ${ }^{30,31}$ the experimental spectra were represented by the superposition of Lorentzians:

$$
I_{\mathrm{VH}}(\omega)=\int_{0}^{\infty} \rho(\Gamma) \frac{\Gamma}{\Gamma^{2}+\omega^{2}} \mathrm{~d} \Gamma
$$

where $\rho(\Gamma)$ is the relaxation rate distribution function. Figure $4 \mathrm{~b}$ displays the spectrum $I_{\mathrm{VH}}(\omega)$ of Figure $4 \mathrm{a}$ in a double logarithmic plot that emphasizes the wings of $I_{\mathrm{VH}}{ }^{-}$ $(\omega)$. The solid line is the computed $I_{\mathrm{VH}}(\omega)$ using the obtained $\rho(\Gamma)$ shown in the inset of Figure $4 \mathrm{~b}$. The presence of the main broad peak in $\rho(\Gamma)$ should have been expected from the failure of the single Lorentzian fit of Figure 4 a which would lead to a $\delta(\Gamma)$ peak in $\rho(\Gamma)$. An unexpected finding is the small peak in $\rho(\Gamma)$ which nevertheless can account for the subtle change of the slope of $\log I_{\mathrm{VH}}(\omega)$ vs $\log \omega$ at high frequencies (Figure $4 \mathrm{~b}$ ). The main peak of $\rho(\Gamma)$ is the continuation of the main relaxation of Figure 3 at high temperatures. On the other hand, the present fast process in the nanosecond time scale is slower compared to the fast process in the picosecond time range recently observed by $\mathrm{DRS}^{7}$ and quasi-elastic neutron scattering. The analysis of the $I_{\mathrm{VH}}(\omega)$ spectrum for the $80 \% \mathrm{PMPS} / \mathrm{CCl}_{4}$ system revealed a similar bimodal structure for $\rho(\Gamma)$ where the second fast peak became faster than in that bulk PMPS.

\section{Concluding Remarks}

The following major conclusions may be drawn from the present study.

(i) The cooperative relaxation phenomenon, which is the basis of stretched exponential behavior, does not necessarily originate from intermolecular correlations. Instead, in the present study, it is demonstrated that shortrange intramolecular coupling might be as well responsible for the apparent nonexponential decay of correlation functions.

(ii) Markov chain models in which bond interdependence is restricted to first neighbors only along the chain are capable of exhibiting $\mathrm{KWW}$ behavior in the intermediate time regime.

(iii) In spite of the large number of differences between the models, conformational correlation functions obey same qualitative behavior. Irrespective of the details of the type and mechanism of relaxation processes, two common features in all three models seem mainly responsible for these observations: the chain connectivity and the short-range intramolecular coupling. (iv) Experimental evidence for the role of intramolecular effects comes from the observation of the $\mathrm{KWW}$ behavior in diluted systems and/or at high temperatures well above the $T_{\mathrm{g}}$ in which intermolecular effects are negligibly small. Present PCS measurements and DRS results by a tandem Fabry-Perot interferometer on poly(methylphenylsiloxane) lend support to the importance of intramolecular correlations.

Acknowledgment. G.F. acknowledges partial support of the EC Network and Mobility Program under Grant No. 114318. I.B. and B.E. acknowledge partial support from Bogazici University Research Fund No. 94P0002.

\section{References and Notes}

(1) Ngai, K. L.; Mashimo, S.; Fytas, G. Macromolecules 1988, 21, 3030.

(2) Boese, D.; Momper, B.; Meier, G.; Kremer, F.; Hagenah, J. U.; Fischer, E. W. Macromolecules 1989, 22, 4416.

(3) Boese, D.; Kremer, F. Macromolecules 1990, 23, 829

(4) Bahar, I.; Erman, B.; Kremer, F.; Fischer, E. W. Macromolecules $1992,25,816$

(5) Williams, G. Adv. Polym. Sci. 1979, 33, 59.

(6) Floudas, G.; Higgins, J. S.; Fytas, G. J. Chem. Phys. 1992, 96 , 7672 .

(7) Steffen, W.; Patkowski, A.; Meier, G.; Fischer, E. W. J. Chem. Phys. 1992, 96, 4171

(8) Fytas, G.; Wang, C. H.; Lilge, D.; Dorfmüller, T.J. Chem. Phys. $1981,75,4247$.

(9) Fytas, G.; Dorfmüller, T.; Wang, C. H. J. Chem. Phys. 1983, 87, 5041.

(10) Fischer, E. W.; Becker, C.; Hagenah, J. U.; Meier, G. Macromolecules $1989,22,4416$.

(11) Johari, G. P.; Goldstein, M. J. Chem. Phys. 1970, 53, 2372.

(12) Fytas, G.; Patkowski, A. In Dynamic Light Scattering; Brown, W., Ed.; Oxford University Press: Oxford, U.K., 1993.

(13) Hyde, P. D.; Ediger, M. Macromolecules 1989, 22, 2253.

(14) Shore, J. E.; Zwanzig, R. J. Chem. Phys. 1975, 63, 5445.

(15) Glauber, R. J. J. Math Phys. 1963, 4, 294.

(16) Hall, C. K.; Helfand, E. J. Chem. Phys. 1982, 77, 3275.

(17) Bahar, I.; Erman, B. Macromolecules 1987, 20, 1368.

(18) Oppenheim, I.; Shuler, K. E.; Weiss, G. H. J. Chem. Phys. 1967, 46,4100

(19) Cook, R.; Helfand, E. J. Chem. Phys. 1985, 82, 1599.

(20) Bahar, I.; Erman, B. Macromolecules 1987, 20, 2310.

(21) Bahar, I.; Erman, B.; Monnerie, L. Macromolecules 1989, 22 , 2396.

(22) Bahar, I.; Mattice, W. L. Macromolecules 1991, 24, 877.

(23) Bahar, I. J. Chem. Phys. 1989, 91, 6525.

(24) Flory, P. J. Statistical Mechanics of Chain Molecules; Interscience: New York, 1969.

(25) Ngai, K. L.; Plazek, D.; Floudas, G. Manuscript in preparation.

(26) Matsuoka, S. Relaxation Phenomena in Polymers; Hanser: Munich, 1992.

(27) Higashigaki, Y.; Wang, C. H. J. Chem. Phys. 1981, 74, 3175.

(28) Fischer, E. W. Physica A 1993, 201, 183.

(29) Dixon, P. K.; Wu, L.; Nagel, S. R.; Williams, B. D.; Carini, J. Phys. Rev. Lett. 1990, 65, 1108.

(30) Alvarez, F.; Colmenero, J.; Kanetakis, J.; Fytas, G. Phys. Rev $B$., in press.

(31) Seils, F.; Steffen, W.; Patkowski, A. Manuscript in preparation. 